\title{
Dimensional reduction without continuous extra dimensions
}

Chamseddine, A H ; Fröhlich, J ; Schubnel, B ; Wyler, D

\begin{abstract}
We describe a novel approach to dimensional reduction in classical field theory. Inspired by ideas from noncommutative geometry, we introduce extended algebras of differential forms over spacetime, generalized exterior derivatives, and generalized connections associated with the "geometry" of space-times with discrete extra dimensions. We apply our formalism to theories of gauge- and gravitational fields and find natural geometrical origins for an axion- and a dilaton field, as well as a Higgs field.
\end{abstract}

DOI: https://doi.org/10.1063/1.4771877

Posted at the Zurich Open Repository and Archive, University of Zurich ZORA URL: https://doi.org/10.5167/uzh-90645

Journal Article

Originally published at:

Chamseddine, A H; Fröhlich, J; Schubnel, B; Wyler, D (2013). Dimensional reduction without continuous extra dimensions. Journal of Mathematical Physics, 54(1):012302.

DOI: https://doi.org/10.1063/1.4771877 


\title{
Dimensional Reduction without Continuous Extra Dimensions
}

\author{
Ali H. Chamseddine \\ American University of Beirut, Physics Department, Beirut, Lebanon and \\ and I.H.E.S. F-91440 Bures-sur-Yvette, France \\ J. Fröhlich, B. Schubnel \\ ETHZ, Mathematics and Physics Departments, Zürich, Switzerland \\ D. Wyler \\ Inst. of Theoretical Physics, University of Zürich, Switzerland
}

\begin{abstract}
We describe a novel approach to dimensional reduction in classical field theory. Inspired by ideas from noncommutative geometry, we introduce extended algebras of differential forms over space-time, generalized exterior derivatives and generalized connections associated with the "geometry" of space-times with discrete extra dimensions. We apply our formalism to theories of gauge- and gravitational fields and find natural geometrical origins for an axionand a dilaton field, as well as a Higgs field.
\end{abstract}




\section{INTRODUCTION}

Introducing extra dimensions in order to unify physical laws and identify natural geometrical origins of various gauge- and scalar fields has quite a long history, beginning in the 1920's with attempts by Kaluza and Klein (see [11], [13]) to unify Maxwell's theory with general relativity in a fivedimensional space-time, continuing with Pauli's construction of non-abelian SU(2)-gauge fields in a six dimensional space-time and culminating with string- and M-theory; (see, e.g., [15]). All these attempts are plagued with the appearance of infinite towers of modes of ever larger mass. In theories where all modes are coupled to the gravitational field such towers may seem to be a problem.

Within the general framework of noncommutative geometry, Connes has proposed to consider generalized notions of differential geometry to describe extra dimensions and to construct classical field theories where certain scalar fields, such as the Higgs field of the standard model, appear for geometrical reasons, but towers of very massive modes do not arise; see [6], [7]. Connes' attempts are based on generalizations of spin geometry. The fundamental geometrical data are encoded in socalled "spectral triples", $(\mathcal{A}, D, \mathcal{H})$, where $\mathcal{A}$ is a (possibly non commutative) *algebra of operators represented on a separable Hilbert space $\mathcal{H}$, and $D$ is an elliptic operator acting on $\mathcal{H}$ generalizing the Dirac operator.

In this note, we present an alternative approach to "dimensional reduction", based on certain extensions of the graded differential algebra, $\Omega(M)$, of differential forms over space-time $\mathrm{M}$, that does not involve introducing continuous extra dimensions, but involves generalized notions of "exterior derivative", "connection" and "metric". Our approach is inspired by Connes' ideas ([6],[7]), but we attempt to generalize general Riemannian - rather than spin-geometry; (see [9]). Thus, besides a *algebra of operators, it involves two anti-commutaing Kähler-Dirac operators, $\mathcal{D}$ and $\overline{\mathcal{D}}$, acting on a Hilbert space of generalized differential forms (rather than a single Dirac operator acting on a Hilbert space of generalized spinors). Classical fields are identified with elements of a (sub-)space of "zero modes"on which $\mathcal{D}^{2}=\overline{\mathcal{D}}^{2}$. The linear combinations $d:=\mathcal{D}-i \overline{\mathcal{D}}$ and $d^{*}:=\mathcal{D}+i \overline{\mathcal{D}}$ can then be interpreted as generalizations of the exterior derivative and its adjoint; (see [9]).

The purpose of our note is to provide natural geometrical interpretations of various scalar fields, such as an axion-, a dilaton and a Higgs field, using ideas and results from [9]. As in Connes' approach, "space-time" will have the structure of two copies of the usual four-dimensional space-time carrying ( a priori massless) left-handed and right-handed spinors, respectively. This is reminiscent of a fivedimensional generalization of the quantum Hall effect discussed in [10], the extra fifth dimension being treated as a discrete two-point set.

The axion will turn out to be the "fifth" component of the electromagnetic vector potential, the dilaton to be a gravitational degree of freedom associated with the discrete fifth dimension, and the Higgs field will appear as a component of the electroweak gauge field that induces tunneling processes between the two sheets of "space-time"and provides masses to the fermions and to the $\mathrm{W}$ - and $\mathrm{Z}$ gauge bosons, as sketched in figure 1.

Our paper is organized as follows. In section II, we summarize, in a sketchy way, some elements of noncommutative geometry that are needed in subsequent sections. For further details, the reader is referred to [6],[7] and [9]. In section III, we first recover an axion field (section III A) by identifying it with the fifth component of the electromagnetic vector potential. This represents the 
simplest application of our formalism. In section III B, we proceed to generalize the Einstein-Hilbert gravitational action to our two-sheeted space-time and find that this leads to the appearance of a dilaton field. Finally, in section III C, we show how the Higgs field of the electroweak theory finds a natural geometrical interpretation within our formalism. Some additional remarks and conclusions are sketched in section IV.

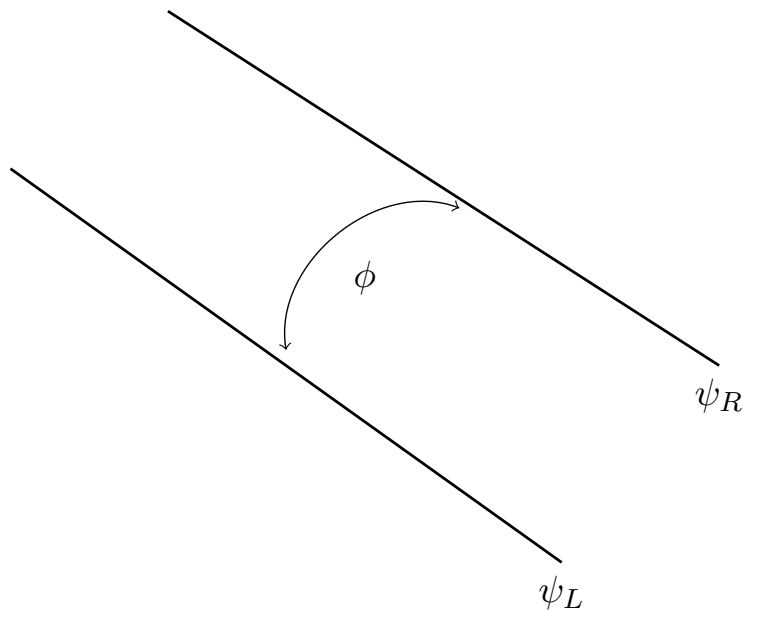

Figure 1: A schematic view of the Yukawa coupling between left- and right-handed fermions, interpreted in a five dimensional space time. The left- and right-handed fermions live on separate four-dimensional sheets. The Higgs field couples left- to right-handed spinors via quantum tunnelling.

\section{GENERALIZED DIFFERENTIAL GEOMETRY}

Gauge theories are intimately related to differential geometry. The reader may remember an undergraduate course on electromagnetism where the Maxwell equations were entirely rewritten in terms of differential forms. Classical fields in a gauge theory with gauge group $G$ are sections of some vector bundles over space-time $M$ associated to a principal $G$-bundle over $M$. Gauge potentials (such as the $\mathrm{U}(1)$ - or $\mathrm{SU}(2)$ - gauge potentials) are $\mathcal{G}$-valued one-forms appearing in the definition of covariant derivatives in a local basis of sections of associated vector bundles, and $\mathcal{G}$ is the Lie algebra of $G$. A well-known theorem of Serre and Swan ([16]) tells us that all finitedimensional vector bundles over a smooth compact manifold $M$ correspond to finitely generated projective $\mathcal{C}^{\infty}(M, \mathbb{C})$-modules. This result motivates our present approach. To generalize classical gauge theories, we will introduce *algebras $\mathcal{A}$ (in particular non-commutative algebras) generalizing the commutative algebra $\mathcal{C}^{\infty}(M, \mathbb{C})$, and then consider finitely generated projective $\mathcal{A}$-modules and define a generalization of the $\mathbb{Z}$-graded algebra of differential forms over $M$. This furnishes the right kind of geometrical data enabling us to generalize the notion of gauge theories.

\section{A. Basic definitions}

Let $\mathcal{A}$ be a unital *algebra over the field $K=\mathbb{R}$ or $\mathbb{C}$. We denote by $\Omega(\mathcal{A})=\bigoplus_{p} \Omega^{p}(\mathcal{A})$ any $\mathbb{Z}$-graded differential algebra with $\mathcal{A}=\Omega^{0}(\mathcal{A})$. The graded product over the "algebra of generalized 
differential forms" $\Omega(\mathcal{A})$ is denoted by $\omega \omega^{\prime}$, where $\omega, \omega^{\prime}$ are elements of $\Omega(\mathcal{A})$. The degree of a homogeneous element $\omega \in \Omega(\mathcal{A})$ is denoted by $\operatorname{deg}(\omega)$.

Definition 1. Vector bundles over $\mathcal{A}$

Inspired by the theorem of Serre and Swan, one defines a noncommutative vector bundle, $\mathcal{M}(\mathcal{A})$, over $\mathcal{A}$ as a finitely generated projective (left) $\mathcal{A}$-module (see [6]). Every such module admits a generating family, i.e., there exist $s_{1}, \ldots, s_{n} \in \operatorname{Hom}(\mathcal{M}(\mathcal{A}), \mathcal{A}), e_{1}, \ldots, e_{n} \in \mathcal{M}(\mathcal{A})$ such that, for all $x \in \mathcal{M}(\mathcal{A})$,

$$
x=\sum_{i=1}^{n} s_{i}(x) e_{i}
$$

The set $\left\{e_{i} \in \mathcal{M}(\mathcal{A}), i=1, \ldots, n \in \mathbb{N}\right\}$, is called a generating family of sections of the vector bundle $\mathcal{M}(\mathcal{A})$.

Next, we assume that there exists a $\mathbb{Z}_{2}$-graded nilpotent operator $d_{\mathcal{A}}\left(d_{\mathcal{A}}^{2}=0\right)$ acting on a $\mathbb{Z}$-graded differential algebra $\Omega(\mathcal{A})$. Since $\Omega(\mathcal{A})$ is a left $\Omega(\mathcal{A})$-module, we may define the differential

$$
\delta_{\mathcal{A}}:=\left[d_{\mathcal{A}}, \cdot\right]_{g}
$$

on the algebra $\Omega(\mathcal{A})$, where the commutator $[\cdot, \cdot]_{g}$ respects the $\mathbb{Z}_{2}$-grading of $\Omega(\mathcal{A})$, i.e.,

$$
\left[d_{\mathcal{A}}, \omega_{p}\right]_{g}=d_{\mathcal{A}} \omega_{p}+(-1)^{p+1} \omega_{p} d_{\mathcal{A}}
$$

for any $\omega_{p}$ of degree $p$. For all homogeneous $\omega \in \Omega(\mathcal{A})$, we may assume that $d_{\mathcal{A}} \omega$ is homogeneous. Note that $\delta_{\mathcal{A}}$ is nilpotent:

$$
\begin{aligned}
\delta_{\mathcal{A}}^{2} \omega & =d_{\mathcal{A}}^{2} \omega+(-1)^{\operatorname{deg}(\omega)+1} d_{\mathcal{A}} \omega d_{\mathcal{A}}+(-1)^{\operatorname{deg}\left(d_{\mathcal{A}} \omega\right)+1} d_{\mathcal{A}} \omega d_{\mathcal{A}}+\omega d_{\mathcal{A}}^{2} \\
& =d_{\mathcal{A}}^{2} \omega+\omega d_{\mathcal{A}}^{2}=0 .
\end{aligned}
$$

Furthermore, $\delta_{A}$ obeys the Leibniz's rule

$$
\delta_{\mathcal{A}}\left(\omega \omega^{\prime}\right)=\delta_{\mathcal{A}}(\omega) \omega^{\prime}+(-1)^{\operatorname{deg}(\omega)} \omega \delta_{\mathcal{A}} \omega^{\prime}
$$

and $\delta_{\mathcal{A}}\left(1_{\mathcal{A}}\right)=0$.

\section{Definition 2. Connections}

Let $\mathcal{M}(\mathcal{A})$ be a projective, finitely generated (left) $\mathcal{A}$-module, and let $\delta_{\mathcal{A}}$ be defined as in (1). A connection, $\nabla$, on $\mathcal{M}(\mathcal{A})$ associated to $\delta_{\mathcal{A}}$ is a $\mathbb{C}$-linear map

$$
\nabla: \mathcal{M}(\mathcal{A}) \longrightarrow \Omega^{\text {odd }}(\mathcal{A}) \otimes_{\mathcal{A}} \mathcal{M}(\mathcal{A})
$$


such that, for all $a \in \mathcal{A}, s \in \mathcal{M}(\mathcal{A})$,

$$
\nabla(a s)=\delta_{\mathcal{A}} a \otimes s+a \nabla s .
$$

$\delta_{\mathcal{A}} a$ in (4) is understood as $\left(\delta_{\mathcal{A}} a\right) 1_{\mathcal{A}}=d_{\mathcal{A}} a-a d_{\mathcal{A}} 1_{\mathcal{A}}$. Every projective finitely generated module having a generating family $\left\{e_{i}\right\}_{i=1}^{n}$ of sections, connections are entirely determined by their action on the $e_{i}$ 's

$$
\nabla\left(e_{i}\right)=-\Omega_{i}^{j} \otimes e_{j}
$$

where $\Omega_{i}^{j} \in \Omega^{\text {odd }}(\mathcal{A})$. The forms $\Omega_{i}^{j}$ correspond to the gauge potential in classical gauge theories. If the module is free and the generating family is a basis, one can choose arbitrary forms $\Omega_{i}^{j}$. If the module is not free one has to impose some restrictions on the coefficients $\Omega_{i}^{j}([6],[4])$.

We require that

$$
\nabla(\omega \otimes s)=\delta_{\mathcal{A}} \omega \otimes s+(-1)^{\operatorname{deg}(\omega)} \omega \nabla s
$$

for all homogeneous $\omega \in \Omega(\mathcal{A}), s \in \mathcal{M}(\mathcal{A})$, where the product is between forms, i.e., $\omega\left(\omega_{1} \otimes s\right)=$ $\left(\omega \omega_{1}\right) \otimes s$. As in $(4), \delta_{\mathcal{A}} \omega$ in $(5)$ is understood as $\left(\delta_{\mathcal{A}} \omega\right) 1_{\mathcal{A}}$. Using $(5)$, we can extend the definition of a connection to $\Omega(\mathcal{A}) \otimes_{\mathcal{A}} \mathcal{M}(\mathcal{A})$ in a unique way and define curvature as follows.

Definition 3. Curvature

The curvature of a connection $\nabla$ is the left $\mathcal{A}$-linear map:

$$
-\nabla^{2}: \mathcal{M}(\mathcal{A}) \longrightarrow \Omega^{\text {even }}(\mathcal{A}) \otimes_{\mathcal{A}} \mathcal{M}(\mathcal{A})
$$

\section{B. Generalization of the algebra of differential forms}

Let $\mathcal{A}$ and $\mathcal{B}$ be unital algebras over the field $K=\mathbb{R}$ or $\mathbb{C}$. We consider $\mathbb{Z}$-graded differential algebras $\Omega(\mathcal{A})$ and $\Omega(\mathcal{B})$, with $\mathcal{A}=\Omega^{0}(\mathcal{A}), \mathcal{B}=\Omega^{0}(\mathcal{B})$. We write $\mathcal{C}:=\mathcal{A} \otimes_{K} \mathcal{B}$. Then $\Omega(\mathcal{A}) \otimes_{K} \Omega(\mathcal{B})$ is a left $\mathcal{C}$-module and can be equipped with a graded product. Henceforth we usually omit the " $K$ " in $\otimes_{K}$.

Definition 4. Graded product over $\Omega(\mathcal{A}) \otimes \Omega(\mathcal{B})$

The graded product, $\wedge$, over the algebra $\Omega(\mathcal{A}) \otimes \Omega(\mathcal{B})$ is defined as follows: For all homogeneous elements $\omega, \omega^{\prime} \in \Omega(\mathcal{A})$ and $\sigma, \sigma^{\prime} \in \Omega(\mathcal{B})$,

$$
(\omega \otimes \sigma) \wedge\left(\omega^{\prime} \otimes \sigma^{\prime}\right)=(-1)^{\operatorname{deg}(\sigma) \operatorname{deg}\left(\omega^{\prime}\right)} \omega \omega^{\prime} \otimes \sigma \sigma^{\prime} .
$$

With this product, $\Omega(\mathcal{A}) \otimes \Omega(\mathcal{B})$ is a $\mathbb{Z}$-graded algebra, and we have that

$$
(\Omega(\mathcal{A}) \otimes \Omega(\mathcal{B}))^{n}=\bigoplus_{p+q=n} \Omega(\mathcal{A})^{p} \otimes \Omega(\mathcal{B})^{q}
$$


where $\Omega^{p}($.$) is the subspace of \Omega($.$) of degree p$.

We assume that there exist $\mathbb{Z}_{2}$-graded nilpotent operators $d_{\mathcal{A}}$ on $\Omega(\mathcal{A})$ and $d_{\mathcal{B}}$ on $\Omega(\mathcal{B})$.

Definition 5. Extension of $\left(d_{\mathcal{A}}, d_{\mathcal{B}}\right)$

An extension of $\left(d_{\mathcal{A}}, d_{\mathcal{B}}\right)$ is a $\mathbb{Z}_{2}$-graded, linear nilpotent operator $\tilde{d}$ acting on the left $\mathcal{C}$-module $\Omega(\mathcal{C}):=\Omega(\mathcal{A}) \otimes \Omega(\mathcal{B})$ that can be written in the form

$$
\tilde{d}=\alpha d_{\mathcal{A}} \otimes 1_{\mathcal{B}}+\beta \Gamma_{\mathcal{A}} \otimes d_{\mathcal{B}}+\sigma
$$

where $\sigma$ is an odd element of $\Omega(\mathcal{C}), \alpha, \beta \in K$, and $\Gamma_{\mathcal{A}}$ is the involution on $\Omega(\mathcal{A})$ defined by

$$
\Gamma_{\mathcal{A}}(\omega)=(-1)^{\operatorname{deg}(\omega)} \omega
$$

for a homogeneous $\omega \in \Omega(\mathcal{A})$.

As in $(1)$, we define a differential $\tilde{\delta}:=[\tilde{d}, \cdot]_{g}$ on the graded algebra $\Omega(\mathcal{C})$, as well as connections and curvature on any (noncommutative) vector bundle $\mathcal{M}(\mathcal{C})$. When $\sigma=0$ in (8), it is easy to check that $\tilde{d}^{2}=0$. Let $\kappa:=\omega \otimes \omega^{\prime} \in \Omega(\mathcal{C})$, with $\omega$ homogeneous. One then has that

$$
\begin{aligned}
\tilde{d}^{2} \kappa & =\tilde{d}\left(\alpha d_{\mathcal{A}} \omega \otimes \omega^{\prime}+\beta(-1)^{\operatorname{deg}(\omega)} \omega \otimes d_{\mathcal{B}} \omega^{\prime}\right) \\
& =\alpha^{2} d_{\mathcal{A}}^{2} \omega \otimes \omega^{\prime}+\alpha \beta(-1)^{\operatorname{deg}\left(d_{\mathcal{A}} \omega\right)} d_{A} \omega \otimes d_{\mathcal{B}} \omega^{\prime}+\alpha \beta(-1)^{\operatorname{deg}(\omega)} d_{\mathcal{A}} \omega \otimes d_{B} \omega^{\prime}+\beta^{2} \omega \otimes d_{\mathcal{B}}^{2} \omega^{\prime} \\
& =0 .
\end{aligned}
$$

If $\sigma \neq 0$ one must add the conditions that $\left[\alpha d_{\mathcal{A}} \otimes 1_{\mathcal{B}}+\beta \Gamma_{\mathcal{A}} \otimes d_{\mathcal{B}}, \sigma\right]_{g}=0$ and $\sigma^{2}=0$.

Below, we will choose for $\Omega(\mathcal{B})$ the exterior algebra of a finite-dimensional vector space $V$ over $K$, which we denote by $\mathcal{G}(V) ;(\mathcal{G}$ stands for "Grassmann Algebra"). This is a graded commutative algebra over the field $K$. The algebra $\mathcal{B}$ is the field $K$. We denote by $\times$ the exterior product on $\mathcal{G}(V)$, and, with $\mathcal{C}=\mathcal{A} \otimes_{K} K \approx \mathcal{A}, \Omega(\mathcal{A})_{V}:=\Omega(\mathcal{C})=\Omega(\mathcal{A}) \otimes \mathcal{G}(V)$.

Let $\xi_{p} \in \mathcal{G}(V)$ be a homogeneous element of odd degree $p$. The operator $d_{\mathcal{B}}:=\xi_{p} \times($.$) acting on$ $\mathcal{G}(V)$ is linear, $\mathbb{Z}_{2}$-graded and nilpotent. For any $\alpha, \beta \in K$,

$$
\tilde{d}:=\alpha d_{\mathcal{A}} \otimes 1+\beta \Gamma_{\mathcal{A}} \otimes\left(\xi_{p} \times .\right)=\alpha d_{\mathcal{A}} \otimes 1+\beta\left(1_{\mathcal{A}} \otimes \xi_{p}\right) \wedge .
$$

is linear, $\mathbb{Z}_{2}$-graded and nilpotent. More generally, we have the following proposition.

Proposition 1. Let $\xi_{p} \in \mathcal{G}(V)$ be a homogeneous element of odd degree, and, let $\omega \in \Omega(\mathcal{A})$ be an even differential form such that $\delta_{\mathcal{A}}(\omega)=0$. Then, for all $\alpha \in K$,

$$
\tilde{d}=\alpha d_{\mathcal{A}} \otimes 1+\left(\omega \otimes \xi_{p}\right) \wedge .
$$


is a linear nilpotent $\mathbb{Z}_{2}$-graded operator on $\Omega(\mathcal{A})_{V}$.

If $\omega \in Z(\Omega(\mathcal{A})$ ) (the center of $\Omega(\mathcal{A})$ ) and if the vector space $V$ is one dimensional, then $\tilde{\delta}=$ $\left[d_{\mathcal{A}} \otimes 1+\left(\omega \otimes \xi_{1}\right) \wedge, .\right]_{g}$ maps $\Omega(\mathcal{A}) \otimes 1$ to itself,

$$
\tilde{\delta}\left(\omega^{\prime} \otimes 1\right)=\delta_{\mathcal{A}} \omega^{\prime} \otimes 1
$$

for any $\omega^{\prime} \in \Omega(\mathcal{A})$. The action of $\tilde{\delta}$ on $\Omega(\mathcal{A}) \otimes \xi_{1}$ is also of the form $\tilde{\delta}\left(\omega^{\prime} \otimes \xi_{1}\right)=\delta_{\mathcal{A}} \omega^{\prime} \otimes \xi_{1}$. In other words, $\tilde{\delta}=\delta_{\mathcal{A}} \otimes 1$.

Corollary 1. Let $\omega_{i}, i=1, \ldots, n$, be commuting differential forms of homogeneous even degree in $\Omega(\mathcal{A})$ such that $\delta\left(\omega_{i}\right)=0$, and let $\xi_{p_{i}} \in \mathcal{G}(V)$ be homogeneous elements of odd degree. Then, for all $\alpha \in K$,

$$
\tilde{d}=\alpha d_{\mathcal{A}} \otimes 1+\sum_{i}\left(\omega_{i} \otimes \xi_{p_{i}}\right) \wedge
$$

is a linear nilpotent $\mathbb{Z}_{2}$-graded operator on $\Omega(\mathcal{A})_{V}$.

\section{Hermitian structure on $\Omega(\mathcal{A})_{V}$}

Until now, the algebras $\mathcal{A}$ and $\Omega(\mathcal{A})$ have been quite general. From now on, we focus on the case where $\mathcal{A}=\mathcal{C}^{\infty}\left(M, M_{n}(K)\right)$ and $\Omega(\mathcal{A})=\Omega\left(M, M_{n}(K)\right)$ is the $\mathbb{Z}$-graded algebra of $M_{n}(K)$ ( $n \times n$ matrices) valued forms, where $M$ is a compact, orientable, smooth manifold of dimension $m$. In this section, we suppose that $M$ is Riemannian. The exterior derivative on $\Omega\left(M, M_{n}(K)\right)$ is denoted by $d$. We construct a hermitian structure on $\Omega^{p}(\mathcal{A})_{V}$ using a generalization of the Hodge operator on $\Omega(\mathcal{M})$. Let $\operatorname{dim}(V)=k$. We choose a basis $\left(\xi^{m+1}, \ldots, \xi^{m+k}\right)$ of $V$. This basis has the same properties as the fermionic superspace coordinates used in the theory of supermanifolds. We introduce the notion of Berezin integration on $\mathcal{G}(V)$ well known from fermionic functional integrals.

Definition 6. Berezin integration

Let $\int_{b}$ denote Berezin integration on $\mathcal{G}(V)$, i.e., $\int_{b} d \xi^{i} \xi^{i}=1, \int_{b} d \xi^{i}=0$, and

$$
\int_{b} d \xi^{m+k} \ldots d \xi^{m+1} \xi^{m+1} \ldots \xi^{m+k}=1
$$

Take $\left(d x^{1}, \ldots, d x^{m}\right)$ to be a coordinate basis of 1 -forms on $M$. To define the extended Hodge operator, we write: $\xi^{i}:=d x^{i} \otimes 1 \equiv d x^{i}$. The metric on the manifold $\mathrm{M}$ is denoted by $g$. To raise the indices of the totally antisymmetric tensor $\epsilon_{\mu \nu \ldots}$, we extend $g$ by imposing $g^{(m+i) j}=g^{j(m+i)}=$ $\delta^{(m+i) j}$, for all $i \in\{1, \ldots, k\}$ and $j \in\{1, \ldots, m+k\}$. This choice is consistent because it is not affected by any change of coordinates on $M$. 
Definition 7. Extended Hodge operator

The extended Hodge $*$-operator is the map $* .: \Omega^{p}(\mathcal{A})_{V} \longrightarrow \Omega^{m+k-p}(\mathcal{A})_{V}$ defined by:

$$
*\left(\xi^{\mu_{1}} \wedge \ldots \wedge \xi^{\mu_{p}}\right)=\frac{\sqrt{|g|}}{(m+k-p) !} \epsilon_{\nu_{p+1} \ldots \nu_{m+k}}^{\mu_{1} \ldots \mu_{p}} \xi^{\nu_{p+1}} \wedge \ldots \wedge \xi^{\nu_{m+k}}
$$

and if $\omega=\frac{1}{p !} \omega_{\mu_{1} \ldots \mu_{p}} \xi^{\mu_{1}} \wedge \ldots \wedge \xi^{\mu_{p}} \in \Omega^{p}(\mathcal{A})_{V}$, by

$$
*(\omega)=\frac{\sqrt{|g|}}{(m+k-p) ! p !}\left(\omega_{\mu_{1} \ldots \mu_{p}}\right)^{\dagger} \epsilon_{\nu_{p+1} \ldots \nu_{m+k}}^{\mu_{1} \ldots \mu_{p}} \xi^{\nu_{p+1}} \wedge \ldots \wedge \xi^{\nu_{m+k}}
$$

where $^{\dagger}$ is the adjoint on $M_{n}(K)$.

Next, we construct a hermitian structure $(\cdot, \cdot)$ on the $\mathcal{A}$-module $\Omega^{p}(\mathcal{A})_{V}$, for any $p \in \mathbb{N}$. A hermitian structure is a sesquilinear form $(\cdot, \cdot): \Omega^{p}(\mathcal{A})_{V} \times \Omega^{p}(\mathcal{A})_{V} \rightarrow \mathcal{A}$, such that

$$
\begin{aligned}
& \text { i) }\left(a s, b s^{\prime}\right)=a\left(s, s^{\prime}\right) b^{\dagger} \text {, for all } a, b \in \mathcal{A}, s, s^{\prime} \in \Omega^{p}(\mathcal{A})_{V}, \\
& \text { ii) }(s, s) \geq 0 \text {, for all } s \in \Omega^{p}(\mathcal{A})_{V} \text {, and }(s, s)=0 \Rightarrow s=0 .
\end{aligned}
$$

For arbitrary $\omega, \omega^{\prime} \in \Omega^{p}(\mathcal{A})_{V}$, we define $(\cdot, \cdot)$ by

$$
\omega \wedge\left(* \omega^{\prime}\right)=:\left(\omega, \omega^{\prime}\right) d \mathcal{V}
$$

where $d \mathcal{V}=\sqrt{|g|} \xi^{1} \ldots \xi^{m+k}$ is the invariant extended volume form. The fact that $(\cdot, \cdot)$ defined in (16) satisfies properties i) and ii) of (15) is obvious from the definitions.

The space $\Omega^{p}(\mathcal{A})_{V}$ of $p$-forms also carries a scalar product

$$
\begin{aligned}
\langle\cdot, \cdot\rangle: \Omega^{p}(\mathcal{A})_{V} \times \Omega^{p}(\mathcal{A})_{V} & \longrightarrow \mathbb{C} \cup\{ \pm \infty\} \\
\left(\omega, \omega^{\prime}\right) & \longmapsto\left\langle\omega, \omega^{\prime}\right\rangle:=f \operatorname{tr}\left(\omega \wedge *\left(\omega^{\prime}\right)\right),
\end{aligned}
$$

where we have set

$$
f \omega:=(-1)^{m k} \int_{M} \int_{b} d \xi^{m+k} \ldots d \xi^{m+1} \omega
$$

The factor $(-1)^{m k}$ ensures positivity of the scalar product and comes from the anticommutation relations $\left\{\xi^{i}, \xi^{j}\right\}=0$, for $i, j \in\{1, \ldots, m+k\}$. On the right-hand side, the Berezin integration is defined in the following way: For all $\omega=\frac{1}{p !} \omega_{\mu_{1} \ldots \mu_{p}} \xi^{\mu_{1}} \ldots \xi^{\mu_{p}} \in \Omega^{p}(\mathcal{A})_{V}$,

$$
\int_{b} d \xi^{m+k} \ldots d \xi^{m+1} \omega:=\frac{1}{p !} \omega_{\mu_{1} \ldots \mu_{p}} \int_{b} d \xi^{m+k} \ldots d \xi^{m+1} \xi^{\mu_{1}} \ldots \xi^{\mu_{p}}
$$


and the Berezin integration is carried out by putting all the Berezin variables $\xi^{\mu_{i}}$ on the left after passing them through the coordinate 1-forms. For instance,

$$
\int_{b} d \xi^{1} d x d y \xi^{1}=\left(\int_{b} d \xi^{1} \xi^{1}\right) d x d y=d x d y
$$

\section{DIMENSIONAL REDUCTION}

In this section, we apply "generalized differential geometry" to some examples from classical field theory in order to show that various classical fields, such as the axion, acquire a natural geometrical interpretation. We begin with the axion field that has appeared in [10] by dimensional reduction of Maxwell theory, starting from a five-dimensional bulk space-time.

\section{A. Axion field}

To recover the axion field, only a little change of the differential geometric formulation of electromagnetism is necessary. Let $M$ be a compact four-dimensional Lorentzian manifold without boundary. We consider the algebra $\mathcal{A}=\mathcal{C}^{\infty}(M, \mathbb{C})(K=\mathbb{C})$. The new ingredient that makes the axion field appear is the modification of the graded algebra of differential forms over $M$. We choose $V=\left\{\lambda \xi_{1}, \lambda \in \mathbb{C}\right\}$ the one-dimensional vector space spanned by $\xi_{1}$, and its exterior algebra $\mathcal{G}(V)$. On $\Omega(\mathcal{A})_{V}=\Omega(M, \mathbb{C}) \otimes \mathcal{G}(V)$, we define a natural generalization of the exterior derivative satisfying the hypotheses of Proposition 1.

$$
\tilde{d}=d \otimes 1+\alpha\left(1 \otimes \xi_{1}\right) \wedge
$$

with $\alpha \in \mathbb{C}$. A connection $\nabla$ on $\mathcal{M}(\mathcal{A}):=\mathcal{A}=\mathcal{C}^{\infty}(M, \mathbb{C})$ is a $\mathbb{C}$-linear map

$$
\nabla: \mathcal{C}^{\infty}(M, \mathbb{C}) \longrightarrow \Omega^{1}(\mathcal{A})_{V} \otimes_{\mathcal{C}^{\infty}(M)} \mathcal{C}^{\infty}(M, \mathbb{C}) \cong \Omega^{1}(\mathcal{A})_{V}
$$

Proposition 2. Let $\nabla$ be any connection on $\mathcal{C}^{\infty}(M, \mathbb{C})$ and $f \in \mathcal{C}^{\infty}(M, \mathbb{C})$. Then

$$
\nabla f=-\Omega \otimes f
$$

where $\Omega=\omega+\phi \xi_{1}$, with $\omega \in \Omega^{1}(M, \mathbb{C}), \phi \in \mathcal{C}^{\infty}(M, \mathbb{C})$.

This proposition follows directly from the definition of $\nabla$. The module being free, we require that $\phi \in \mathcal{C}^{\infty}(M, \mathbb{R})$, so that the field $\phi$ has zero charge. $\phi$ will turn out to be the axion field. The curvature (see (6)) associated to a connection is

$$
\begin{aligned}
-\nabla^{2} f & =-\nabla(-\Omega \otimes f)=(\tilde{\delta} \Omega) \otimes f-\Omega \wedge \nabla f \\
& =(\tilde{\delta} \Omega) \otimes f=: F_{\nabla^{2}} \otimes f
\end{aligned}
$$


with

$$
F_{\nabla^{2}}=\tilde{\delta} \Omega=\left[\tilde{d}, \omega+\phi \xi_{1}\right]_{+} 1=d \omega+d \phi \xi_{1}
$$

In [10], the integral of the Chern-Simons five-form led to an axion term in the action. The corresponding extended integral of the extended Chern-Simons five-form $\Omega \wedge F_{\nabla^{2}} \wedge F_{\nabla^{2}}$ is given by

$$
\begin{aligned}
f \Omega \wedge F_{\nabla^{2}} \wedge F_{\nabla^{2}} & =f\left(\omega+\phi \xi_{1}\right) \wedge\left(d \omega+d \phi \xi_{1}\right) \wedge\left(d \omega+d \phi \xi_{1}\right) \\
& =f\left(\omega+\phi \xi_{1}\right)\left((d \omega)^{2}+2 d \omega d \phi \xi_{1}\right) \\
& =f\left(\omega d \omega d \omega+\phi \xi_{1}(d \omega)^{2}+2 \omega d \omega d \phi \xi_{1}\right) .
\end{aligned}
$$

The Berezin integration $\int_{b} d \xi_{1}=0$ implies that $f \omega d \omega d \omega=0$.

$$
\begin{aligned}
f \Omega \wedge F_{\nabla^{2}} \wedge F_{\nabla^{2}} & =f\left(\phi(d \omega)^{2} \xi_{1}+2 \omega d \omega d \phi \xi_{1}\right) \\
& =\int_{M}\left(\phi(d \omega)^{2}+2 \omega d \omega d \phi\right) .
\end{aligned}
$$

The manifold $M$ has no boundary, and therefore

$$
0=\int_{M} d(\omega d \omega \phi)=\int_{M} d \omega d \omega \phi-\int_{M} \omega d \omega d \phi
$$

which finally yields

$$
f \Omega \wedge F_{\nabla^{2}} \wedge F_{\nabla^{2}}=3 \int_{M} \phi(d \omega)^{2}
$$

with $d \omega$ in (22) the electromagnetic field strength in four-dimensional space-time. We see that $\phi$ can be interpreted as an axion field that couples to the electromagnetic field. We find the same result as in [10]. However, we have not added any extra continuous dimension. We recover the kinetic term for the axion by dimensional reduction of the Maxwell action

$$
\begin{aligned}
f F_{\nabla^{2}} \wedge\left(* F_{\nabla^{2}}\right) & =\int_{M} d \omega \wedge *(d \omega)_{4}+f\left(d \phi \xi_{1}\right) \wedge *\left(d \phi \xi_{1}\right) \\
& =\int_{M} d \omega \wedge *(d \omega)_{4}+\int_{M} \partial^{\mu} \phi \partial_{\mu} \phi \sqrt{|g|} d^{4} x
\end{aligned}
$$

where $*(.)_{4}$ is the Hodge operator on $\Omega(M)$.

\section{B. Gravity with dilaton}

We derive an Einstein-Hilbert action with dilaton using our formalism. We consider a fourdimensional compact Lorentzian manifold $M$ without boundary and choose $K=\mathbb{R}, \mathcal{A}=\mathcal{C}^{\infty}(M, \mathbb{R})$ 
and $V=\left\{\lambda \xi_{1}, \lambda \in \mathbb{R}\right\}$. On $\Omega(\mathcal{A})_{V}:=\Omega(M, \mathbb{R}) \otimes \mathcal{G}(V)$, we take

$$
\tilde{d}=d \otimes 1+\alpha\left(1 \otimes \xi_{1}\right) \wedge \cdot
$$

We consider the vector bundle $\mathcal{M}(\mathcal{A})=\Omega^{1}(\mathcal{A})_{V}$. It generalizes the cotangent bundle of the manifold $M$. Connections, $\nabla$, on $\mathcal{M}(\mathcal{A})$ are linear maps:

$$
\nabla: \Omega^{1}(\mathcal{A})_{V} \longrightarrow \Omega^{1}(\mathcal{A})_{V} \otimes_{\mathcal{A}} \Omega^{1}(\mathcal{A})_{V}
$$

To keep our notation simple in the following calculations, we identify $\xi_{1} \equiv d x^{4}$, as if $\xi_{1}$ were the coordinate one-form corresponding to an extra dimension. We introduce an extension of the Cartan basis

$$
E^{A}=e_{C}^{A} d x^{C}
$$

where $A, C=0, \ldots, 4$ and $\left(E^{A}, E^{B}\right)=\eta^{A B} ;(\cdot, \cdot)$ is the hermitian structure on $\Omega^{1}(\mathcal{A})_{V}$ defined in (16), and $\eta^{A B}$ is the Minkowski metric tensor in five dimensions with signature $(-,+,+,+,+)$.

Proposition 3. Let $\nabla$ be a connection on $\Omega^{1}(\mathcal{A})_{V}$. With respect to the Cartan basis,

$$
\nabla E^{A}=-\Omega_{B}^{A} \otimes E^{B}
$$

where $\Omega_{B}^{A} \in \Omega^{1}(M, \mathbb{R})$, for $A, B \in\{0, \ldots, 4\}$, i.e.,

$$
\Omega_{B}^{A}=\omega_{B}^{A}+\phi_{B}^{A} d x^{4}
$$

with $\omega_{B}^{A} \in \Omega^{1}(\mathcal{A})_{V}, \phi_{B}^{A} \in \mathcal{C}^{\infty}(M, \mathbb{R})$.

The curvature two form associated to $\nabla$ takes the form:

$$
\begin{aligned}
-\nabla^{2}\left(\alpha_{A} E^{A}\right) & =-\nabla\left(\left(\tilde{\delta} \alpha_{A}\right) \otimes E^{A}-\alpha_{A} \Omega_{B}^{A} \otimes E^{B}\right) \\
& =-\left[\left(\tilde{\delta} \alpha_{A} \wedge \Omega_{B}^{A}\right) \otimes E^{B}-\tilde{\delta}\left(\alpha_{A} \Omega_{B}^{A}\right) \otimes E^{B}-\alpha_{A} \Omega_{B}^{A} \wedge \Omega_{C}^{B} \otimes E^{C}\right] \\
& =\alpha_{A}\left(\tilde{\delta} \Omega_{C}^{A}+\Omega_{B}^{A} \wedge \Omega_{C}^{B}\right) \otimes E^{C}=\alpha_{A} \mathcal{R}_{C}^{A} \otimes E^{C}
\end{aligned}
$$

where

$$
\mathcal{R}_{C}^{A}=\tilde{\delta} \Omega_{C}^{A}+\Omega_{B}^{A} \wedge \Omega_{C}^{B}
$$

We can compute the scalar curvature using (27). In the following calculations, we denote by capital letters $A, B, \ldots$ indices that take values in $\{0,1,2,3,4\}$ and by $a, b, \ldots$ indices in the range 0 to 3 . To simplify matters, we suppose that the Cartan basis is of the form

$$
E^{A}=\delta_{a}^{A} e_{\mu}^{a} d x^{\mu}+\delta_{4}^{A} e^{\sigma} d x^{4}
$$


where $\sigma \in \mathcal{C}^{\infty}(M, \mathbb{R})$. With this ansatz, we tacitly assume that the added dimension does not "warp" when one moves along $M$. The hermitian structure $(\cdot, \cdot)$ defined in (16) satisfies $\left(d x^{\mu}, d x^{4}\right)=$ 0 . We make the following hypotheses:

- The connection is torsion free, i.e., $T(\nabla)=0$; (for the definition of torsion see [9])

- The connection is unitary with respect to the metric on the extended tangent space, i.e.,

$$
\tilde{\delta}\left(\omega_{1}, \omega_{2}\right)=\left(\nabla \omega_{1}, \omega_{2}\right)+\left(\omega_{1}, \nabla \omega_{2}\right)
$$

for arbitrary $\omega_{1}, \omega_{2} \in \Omega^{1}(\mathcal{A})_{V}$.

These constraints characterize the Levi-Civita connection.

\section{Torsion-free condition}

One has that

$$
T(\nabla) E^{A}=\tilde{\delta} E^{A}+\Omega_{B}^{A} \wedge E^{B}=0 .
$$

By writing $e^{a}=e_{\mu}^{a} d x^{\mu}$, it is easy to show that this condition leads to the following identities.

- $A=a$ :

$$
\begin{cases}d e^{a}+\omega_{b}^{a} e^{b} & =0 \\ \omega_{\nu 4}^{a} e^{\sigma}-\phi_{b}^{a} e_{\nu}^{b} & =0\end{cases}
$$

- $A=4$ :

$$
\begin{cases}\omega_{\mu b}^{4} e_{\nu}^{b}-\omega_{\nu b}^{4} e_{\mu}^{b} & =0 \\ \left(\partial_{\nu} \sigma\right) e^{\sigma}+\omega_{\nu 4}^{4} e^{\sigma}-\phi_{b}^{4} e_{\nu}^{b} & =0\end{cases}
$$

\section{Unitarity condition}

Next, we use the unitarity condition (29)

$$
\begin{aligned}
\tilde{\delta}\left(E^{A}, E^{B}\right) & =\left(\nabla E^{A}, E^{B}\right)+\left(E^{A}, \nabla E^{B}\right) \\
& =-\left(\Omega_{C}^{A} E^{C}, E^{B}\right)-\left(E^{A}, \Omega_{D}^{B} E^{D}\right) \\
& =-\Omega_{C}^{A} \eta^{C B}-\Omega_{D}^{B} \eta^{A D} .
\end{aligned}
$$

By definition,

$$
\tilde{\delta}\left(E^{A}, E^{B}\right)=\tilde{\delta}\left(\eta^{A B}\right)=0 .
$$


Consequently, we are led to

$$
\left\{\begin{array}{l}
\omega_{C}^{A} \eta^{C B}+\omega_{D}^{B} \eta^{A D}=0 \\
\phi_{C}^{A} \eta^{C B}+\eta^{A D} \phi_{D}^{B}=0
\end{array}\right.
$$

Listing all the possibilities for the components $A$ and $B$ of $\omega$ and $\phi$, and using equations (30) and (31), we see that the components of the connection satisfy the identities

$$
\left\{\begin{array}{l}
\phi_{b}^{4}=e_{b}^{\nu}\left(\partial_{\nu} \sigma\right) e^{\sigma} \\
\phi_{4}^{b}=-e_{c}^{\nu}\left(\partial_{\nu} \sigma\right) e^{\sigma} \eta^{c b}
\end{array}\right.
$$

with all other components of $\phi_{B}^{A}$ vanishing. For $\omega_{B}^{A}$, only the forms $\omega_{b}^{a}$ may be non zero.

\section{Components of the curvature tensor}

We have to find an expression for the components of the curvature tensor in terms of the components of the connection calculated in (33). According to (27),

$$
\begin{aligned}
\mathcal{R}_{B}^{A} & =\tilde{\delta} \Omega_{B}^{A}+\Omega_{C}^{A} \Omega_{B}^{C} \\
& =\frac{1}{2} R_{B C D}^{A} E^{C} \wedge E^{D} .
\end{aligned}
$$

An easy identification leads to

$$
\begin{aligned}
& R_{B c d}^{A}=e_{c}^{\mu} e_{d}^{\nu}\left(\partial_{\mu} \omega_{\nu B}^{A}-\partial_{\nu} \omega_{\mu B}^{A}+\omega_{\mu E}^{A} \omega_{\nu B}^{E}-\omega_{\nu E}^{A} \omega_{\mu B}^{E}\right) \\
& R_{B 4 d}^{A}=e^{-\sigma} e_{d}^{\nu}\left(-\partial_{\nu} \phi_{B}^{A}+\phi_{E}^{A} \omega_{\nu B}^{E}-\omega_{\nu E}^{A} \phi_{B}^{E}\right) .
\end{aligned}
$$

As our main goal is to compute the scalar curvature, we have to find the components of the Ricci tensor using that

$$
R_{B D}=R_{B A D}^{A}=R_{B a D}^{a}+R_{B 4 D}^{4}
$$

Because the scalar curvature is given by

$$
R=\eta^{B D} R_{B D}
$$

we only have to determine $R_{b d}$ and $R_{44}$. For instance,

$$
\begin{aligned}
R_{b d} & =R_{b a d}^{a}+R_{b 4 d}^{4} \\
& =\underbrace{e_{a}^{\mu} e_{d}^{\nu}\left(\partial_{\mu} \omega_{\nu b}^{a}-\partial_{\nu} \omega_{\mu b}^{a}+\omega_{\mu c}^{a} \omega_{\nu b}^{c}-\omega_{\nu c}^{a} \omega_{\mu b}^{c}\right)}_{=R_{b d}^{(4)}}+ \\
& +\underbrace{e^{-\sigma} e_{d}^{\nu}\left(-\partial_{\nu} \phi_{b}^{4}+\phi_{c}^{4} \omega_{\nu b}^{c}-\omega_{\nu c}^{4} \phi_{b}^{c}+\phi_{4}^{4} \omega_{\nu b}^{4}-\omega_{\nu 4}^{4} \phi_{b}^{4}\right)}_{(I)} .
\end{aligned}
$$


It is possible to evaluate (I) using properties of the Cartan basis. One finds that

$$
(I)=e_{d}^{\nu}(-\underbrace{\left[\partial_{\nu} e_{b}^{\mu}-\omega_{\nu b}^{c} e_{c}^{\mu}\right]}_{(I I)} \partial_{\mu} \sigma-e_{b}^{\mu}\left(\partial_{\nu} \partial_{\mu} \sigma\right)-e_{b}^{\mu}\left(\partial_{\nu} \sigma\right)\left(\partial_{\mu} \sigma\right)) .
$$

The term (II) underlined above reduces to $(I I)=-e_{b}^{\alpha} \Gamma_{\nu \alpha}^{\mu}$ where $\Gamma_{\nu \alpha}^{\mu}$ are the Christoffel symbols, defined, in any coordinate basis, by

$$
\nabla^{(4)}\left(d x^{\mu}\right)=-\Gamma_{\nu \alpha}^{\mu} d x^{\nu} \otimes d x^{\alpha}
$$

and $\nabla^{(4)}$ is the Levi-Civita connection on $\Omega^{1}(M, \mathbb{R})$, given by

$$
\nabla^{(4)}\left(E^{a}\right)=-\omega_{b}^{a} \otimes E^{b} .
$$

Indeed,

$$
\begin{aligned}
\nabla^{(4)}\left(E^{a}\right) & =\nabla^{(4)}\left(e_{\kappa}^{a} d x^{\kappa}\right)=\partial_{\nu} e_{\kappa}^{a} d x^{\nu} \otimes d x^{\kappa}-e_{\delta}^{a} \Gamma_{\nu \kappa}^{\delta} d x^{\nu} \otimes d x^{\kappa} \\
& =-\omega_{\nu c}^{a} e_{\kappa}^{c} d x^{\nu} \otimes d x^{\kappa}
\end{aligned}
$$

which yields

$$
\partial_{\nu} e_{\kappa}^{a}-e_{\delta}^{a} \Gamma_{\nu \kappa}^{\delta}=-\omega_{\nu c}^{a} e_{\kappa}^{c}
$$

Moreover, as $e_{\mu}^{a} e_{b}^{\mu}=\delta_{b}^{a}$,

$$
\partial_{\nu} e_{b}^{\mu}=-e_{b}^{\kappa} e_{a}^{\mu} \partial_{\nu} e_{\kappa}^{a}
$$

Plugging (38) and (39) into (II),

$$
\begin{aligned}
(I I) & =-e_{b}^{\kappa} e_{a}^{\mu}\left(-\omega_{\nu c}^{a} e_{\kappa}^{c}+e_{\delta}^{a} \Gamma_{\nu \kappa}^{\delta}\right)-\omega_{\nu b}^{c} e_{c}^{\mu} \\
& =-e_{b}^{\alpha} \Gamma_{\nu \alpha}^{\mu}
\end{aligned}
$$

Thus,

$$
(I)=-e_{d}^{\nu} e_{b}^{\mu}(\underbrace{\left[-\Gamma_{\nu \mu}^{\alpha} \partial_{\alpha} \sigma+\partial_{\mu} \partial_{\nu} \sigma\right]}_{=\nabla_{\mu}^{(4)} \partial_{\nu} \sigma}+\left(\partial_{\nu} \sigma\right)\left(\partial_{\mu} \sigma\right))
$$

where we have identified the components of the covariant derivative of $\partial_{\nu} \sigma$. Then,

$$
R_{b d}=R_{b d}^{(4)}-e_{d}^{\nu} e_{b}^{\mu}\left(\nabla_{\mu}^{(4)} \partial_{\nu} \sigma+\left(\partial_{\nu} \sigma\right)\left(\partial_{\mu} \sigma\right)\right) .
$$


In the same way, one finds for $R_{44}$

$$
R_{44}=-e_{a}^{\nu} e_{c}^{\mu} \eta^{c a}\left[\nabla_{\nu}^{(4)} \partial_{\mu} \sigma+\left(\partial_{\nu} \sigma\right)\left(\partial_{\mu} \sigma\right)\right]
$$

Using (37), the extended scalar curvature is given by

$$
R^{(5)}=R^{(4)}-2 g^{\mu \nu}\left[\nabla_{\nu}^{(4)} \partial_{\mu} \sigma+\left(\partial_{\nu} \sigma\right)\left(\partial_{\mu} \sigma\right)\right] .
$$

\section{Einstein-Hilbert action and Dilaton}

The generalized Einstein-Hilbert action reads

$f \sqrt{|g|} R^{(5)} e^{\sigma} d^{4} x \xi_{1}=\int_{M} d^{4} x \sqrt{|g|} R^{(5)} e^{\sigma}=\int_{M} d^{4} x \sqrt{|g|} e^{\sigma}\left(R^{(4)}-2 g^{\mu \nu}\left[\nabla_{\nu} \partial_{\mu} \sigma+\left(\partial_{\nu} \sigma\right)\left(\partial_{\mu} \sigma\right)\right]\right)$

where we have replaced $\nabla_{\nu}^{(4)}$ by $\nabla_{\nu}$, as there is no risk of confusion, anymore. One can use a conformal transformation to change the form of the integrand. Suppose that we rescale the metric,

$$
\tilde{g}_{\mu \nu}=e^{2 \Phi} g_{\mu \nu}
$$

For a manifold $M$ of dimension $d$ (cf. [5]), this rescaling changes the scalar curvature by

$$
e^{2 \Phi} \tilde{R}-R=-2(d-1) \nabla^{\nu} \partial_{\nu} \Phi-(d-2)(d-1)\left(\partial^{\nu} \Phi\right)\left(\partial_{\nu} \Phi\right) .
$$

$M$ is four-dimensional and if we choose $\Phi=\frac{1}{2} \sigma$, we find that

$$
e^{\sigma} \tilde{R}-R=-3 \nabla^{\nu} \partial_{\nu} \sigma-\frac{3}{2}\left(\partial^{\nu} \sigma\right)\left(\partial_{\nu} \sigma\right)
$$

Here $R=R^{(4)}$. Consequently, the generalized Hilbert-Einstein action is given by

$$
\int_{M} d^{4} x \sqrt{|g|} e^{\sigma} R^{(5)}=\int_{M} d^{4} x \sqrt{|\tilde{g}|}\left(\tilde{R}-\frac{1}{2} \tilde{g}^{\mu \nu}\left(\partial_{\nu} \sigma\right)\left(\partial_{\mu} \sigma\right)+\tilde{\nabla}^{\nu} \partial_{\nu} \sigma\right) .
$$

$\tilde{\nabla}^{\nu} \partial_{\nu} \sigma$ can be rewritten as $\frac{1}{\sqrt{|\tilde{g}|}} \partial_{\nu}\left(\sqrt{|\tilde{g}|} \partial^{\nu} \sigma\right)$. As $M$ is without boundary,

$$
\int_{M} d^{4} x \sqrt{|\tilde{g}|} \tilde{\nabla}^{\nu} \partial_{\nu} \sigma=0
$$

and only the kinetic term for the dilaton remains (cf. for instance [8]):

$$
S=\int_{M} d^{4} x \sqrt{|\tilde{g}|}\left(\tilde{R}-\frac{1}{2} \tilde{g}^{\mu \nu}\left(\partial_{\nu} \sigma\right)\left(\partial_{\mu} \sigma\right)\right) .
$$




\section{Electroweak theory with a Higgs field}

Let $\mathrm{M}$ be a four-dimensional compact Lorentzian manifold without boundary and $\mathcal{A}=\mathcal{C}^{\infty}(M, \mathbb{C})$. We consider the $\mathcal{A}$-bimodule $\tilde{\mathcal{M}}(\mathcal{A})=S^{2}(M) \otimes_{\mathcal{A}} \mathcal{M}(\mathcal{A})$ where $\mathcal{M}(\mathcal{A})=\mathcal{C}^{\infty}\left(M, \mathbb{C}^{2} \oplus \mathbb{C}\right)$ and $S^{2}(M)$ is the Hilbert space of square integrable spinors on $M . \tilde{\mathcal{M}}(\mathcal{A})$ is projective and finitely generated. We consider the one-dimensional vector space $V=\left\{\lambda \xi_{1}, \lambda \in \mathbb{C}\right\}$ and introduce the exterior derivative

$$
\tilde{d}=d \otimes 1
$$

on $\Omega(\mathcal{A})_{V}$. Connections on $\mathcal{M}(\mathcal{A})$ are linear maps

$$
\nabla: \mathcal{M}(\mathcal{A}) \longrightarrow \Omega^{\text {odd }}(\mathcal{A})_{V} \otimes_{\mathcal{A}} \mathcal{M}(\mathcal{A})
$$

Once we have constructed a connection on $\mathcal{M}(\mathcal{A})$, we can construct a connection on $\tilde{\mathcal{M}}(\mathcal{A})$ in the following way. Let $\nabla_{S^{2}}$ be the canonical spin connection on $S^{2}(M)$. We define

$$
\tilde{\nabla}: \tilde{\mathcal{M}}(\mathcal{A}) \longrightarrow \Omega^{\text {odd }}(\mathcal{A})_{V} \otimes_{\mathcal{A}} \tilde{\mathcal{M}}(\mathcal{A})
$$

by

$$
\tilde{\nabla}(\psi \otimes f)=\nabla_{S^{2}} \psi \otimes f+\pi(\psi \otimes \nabla f)
$$

where $\pi(\psi \otimes \omega \otimes f)=\omega \otimes \psi \otimes f$, for all $\psi \in S^{2}(M), \omega \in \Omega(\mathcal{A})_{V}$ and $f \in \mathcal{M}(\mathcal{A})$.

We construct a connection $\nabla$ on the free $\mathcal{A}$-module $\mathcal{M}(\mathcal{A})$. Let $\left(s_{1}, s_{2}, s_{3}\right)$ be a basis of $\mathcal{M}(\mathcal{A})$.

$$
\nabla s_{i}=-\underbrace{\Omega_{i}^{j}}_{\in \Omega^{1}(\mathcal{A})_{V}} \otimes s_{j}
$$

Similarly to (27), the components of the curvature tensor are given by

$$
\left(F_{\Omega}\right)_{j}^{i}=\tilde{\delta} \Omega_{j}^{i}+\Omega_{k}^{i} \Omega_{j}^{k}
$$

The general form of $\Omega:=\left(\Omega_{i}^{j}\right)$ reads, in matrix notation,

$$
\Omega=A \otimes 1+B \otimes \xi_{1}
$$

where $A \in \Omega\left(M, M_{3}(\mathbb{C})\right), B \in M_{3}(\mathbb{C})$. The module being free, we can take an arbitrary consistent choice for $A, B$ in (47). We first introduce a Hermitian structure on $\mathcal{M}(\mathcal{A})$ in which the basis is orthonormal, i.e., we choose $(\cdot, \cdot)$ such that $\left(s_{i}, s_{j}\right)=\delta_{i j}$ and $\left(f, f^{\prime}\right)=\sum_{i=1}^{3} f_{i} \bar{f}_{i}^{\prime},(\bar{\cdot})$ denoting complex conjugation. We require $\Omega$ to be unitary with respect to this metric, i.e., $\Omega$ must be skew-hermitian. We would like $A$ to be chosen as in the Standard Model of particle physics (see, 
e.g. [17]); i.e.,

$$
A=\left(\begin{array}{cc}
\omega_{2 \times 2} & 0_{2 \times 1} \\
0_{1 \times 2} & \alpha_{1 \times 1}
\end{array}\right)
$$

where $\omega=\omega_{\mu} d x^{\mu}$ and $\alpha_{1 \times 1}=\alpha_{\mu} d x^{\mu}$ are the $U(2)$ and $U(1)$ gauge potentials, respectively. The form $\Omega$ being skew-hermitian, $\omega_{\mu}$ must be skew-hermitian and $\alpha_{\mu} \in i \mathbb{R}$. We would like $B \notin M_{2}(\mathbb{C}) \oplus \mathbb{C}$ to exchange left- and right-handed spinors, describing tunneling processes between the two sheets of space-time as explained in section I.

$$
B=\left(\begin{array}{cc}
0_{2 \times 2} & H \\
-H^{\dagger} & 0_{1 \times 1}
\end{array}\right)
$$

where $H \in \mathcal{C}^{\infty}\left(M, M_{2 \times 1}(\mathbb{C})\right)$. We can add to $B$ an axion field $\phi \in \mathcal{C}^{\infty}(M, \mathbb{R})$, as considered in section III A. Then the final form for $\Omega$ is given by

$$
\Omega=\left(\begin{array}{cc}
\omega_{2 \times 2} & 0_{2 \times 1} \\
0_{1 \times 2} & \alpha_{1 \times 1}
\end{array}\right) \otimes 1+\left(\begin{array}{cc}
i \phi 1_{2 \times 2} & H \\
-H^{\dagger} & i \phi
\end{array}\right) \otimes \xi_{1}
$$

Next, we determine the components of the curvature two-form. Before doing so, we propose to investigate how the components of $\Omega$ transform under a gauge transformation.

\section{Gauge transformations}

Consider two bases of sections, $\left\{s_{i}^{\prime}\right\},\left\{s_{j}\right\}$ such that

$$
s_{i}^{\prime}=g_{i}^{j} s_{j}
$$

One has that

$$
\left(\Omega^{\prime}\right)_{i}^{l}=-\left(\tilde{d} g_{i}^{k}\right)\left(g^{-1}\right)_{k}^{l}+g_{i}^{j} \Omega_{j}^{k}\left(g^{-1}\right)_{k}^{l} .
$$

The matrix-valued function $g$ maps a basis of sections to another basis of sections. Since eft- and right-handed spinors should not be mixed by gauge transformations, the most general form for $g$ is

$$
g=\left(\begin{array}{cc}
A_{2 \times 2} & 0 \\
0 & e^{i \theta}
\end{array}\right)
$$


where $A \in U(2), \theta \in \mathbb{R}$. One then finds that

$$
\begin{aligned}
\omega & \rightarrow A \omega A^{\dagger}-d A A^{\dagger} \\
\alpha & \rightarrow \alpha-i d \theta \\
H & \rightarrow A H e^{-i \theta} \\
\phi & \rightarrow \phi .
\end{aligned}
$$

$H$ transforms as the standard Higgs field under a gauge transformation. We will need these formulas to check that the gauge field strength transforms correctly under gauge transformations, i.e.,

$$
F_{\Omega} \rightarrow g F_{\Omega} g^{-1}
$$

\section{Curvature 2-form}

We use the notations

$$
\begin{aligned}
D H & :=d H+\omega H-\alpha H \\
F_{\omega} & :=d \omega+\omega^{2} \\
F_{\alpha} & :=d \alpha .
\end{aligned}
$$

Then

$$
F_{\Omega}=\left(\begin{array}{cc}
F_{\omega}+i(d \phi) \xi_{1} 1_{2 \times 2} & (D H) \xi_{1} \\
-(D H)^{\dagger} \xi_{1} & i d \phi \xi_{1}+F_{\alpha}
\end{array}\right)
$$

Under a gauge transformation $g, F_{\Omega}$ given in (50) satisfies the transformation law

$$
F_{\Omega} \rightarrow\left(\begin{array}{cc}
A_{2 \times 2} & 0 \\
0 & e^{i \theta}
\end{array}\right) F_{\Omega}\left(\begin{array}{cc}
A_{2 \times 2}^{\dagger} & 0 \\
0 & e^{-i \theta}
\end{array}\right)
$$

Indeed,

$$
\begin{aligned}
D H & \rightarrow d\left(A H e^{-i \theta}\right)+\left(A \omega A^{\dagger}-d A A^{\dagger}\right)\left(A H e^{-i \theta}\right)-(\alpha-i d \theta)\left(A H e^{-i \theta}\right) \\
& =d A H e^{-i \theta}+e^{-i \theta} A d H-i A e^{-i \theta} d \theta+A \omega H e^{-i \theta}-d A H e^{-i \theta}-\alpha A H e^{-i \theta}+\overline{i(d \theta) A H e^{-i \theta}} \\
& =A D H e^{-i \theta} .
\end{aligned}
$$

All the other components are easily determined. It follows from (51) that the action functional

$$
S=f \operatorname{tr}\left[F_{\Omega} \wedge\left(* F_{\Omega}\right)\right]
$$

is gauge-invariant. 


\section{Yukawa coupling and kinetic energy term for the Higgs field}

It is not difficult to compute the Hodge dual of $F_{\Omega}$ in the basis $\left(d x^{0}, d x^{1}, d x^{2}, d x^{3}, d x^{4}=\xi_{1}\right)$. If $\omega=\frac{1}{p !} \omega_{\mu_{1} \ldots \mu_{p}} d x^{\mu_{1}} \wedge \ldots \wedge d x^{\mu_{p}} \in \Omega^{p}\left(M, M_{3}(\mathbb{C})\right)_{V}$

$$
*(w)=\frac{\sqrt{|g|}}{(m+1-p) ! p !}\left(\omega_{\mu_{1} \ldots \mu_{p}}\right)^{\dagger} \epsilon_{\nu_{p+1} \ldots \nu_{m+k}}^{\mu_{1} \ldots \mu_{p}} d x^{\nu_{p+1}} \wedge \ldots \wedge d x^{\nu_{m+k}}
$$

As in II C, to raise the lower indices of $\epsilon_{A B \ldots}$, we extend the metric tensor $g$ of the manifold M by defining $g^{4 A}=\delta_{4}^{A}$, for $A \in\{0,1,2,3,4\}$. In what follows, $A, B, \ldots$ are indices that range from 0 to 4 , whereas $\mu, \nu, \ldots$ take values in $\{0,1,2,3\}$. We work in the signature $(-,+,+,+)$ for the Minkowski metric $\eta_{\mu \nu}$.

If the two-form $\omega=\frac{1}{2} \omega_{\mu \nu} d x^{\mu} d x^{\nu}$ does not contain any term with $\xi_{1}$ then

$$
\begin{aligned}
*(\omega) & =\frac{\sqrt{|g|}}{3 ! 2 !}\left(\omega_{\mu \nu}\right)^{\dagger} \epsilon_{A \ldots C}^{\mu \nu} d x^{A} \wedge \ldots \wedge d x^{C} \\
& =\frac{\sqrt{|g|}}{2 ! 2 !}\left(\omega_{\mu \nu}\right)^{\dagger} \epsilon_{\delta \gamma}^{\mu \nu} d x^{\delta} \wedge d x^{\gamma} \wedge \xi_{1} \\
& =*(\omega)_{4} \wedge \xi_{1}
\end{aligned}
$$

where $*(\omega)_{4}$ denotes the Hodge dual on $\Omega\left(M, M_{3}(\mathbb{C})\right)$. We then find that

$$
*\left(F_{\Omega}\right)=\left(\begin{array}{cc}
*\left(F_{\omega}\right)-i\left(\partial_{\mu} \phi\right) *\left(d x^{\mu} \xi_{1}\right) 1_{2 \times 2} & -(D H)_{\mu} *\left(d x^{\mu} \xi_{1}\right) \\
(D H)_{\mu}^{\dagger} *\left(d x^{\mu} \xi_{1}\right) & -i\left(\partial_{\mu} \phi\right) *\left(d x^{\mu} \xi_{1}\right)+*\left(F_{\alpha}\right)
\end{array}\right)
$$

The usual Yang-Mills type action (52) involves only four terms

$$
S=f[(I)+(I I)+(I I I)+(I V)]
$$

where:

$$
\begin{aligned}
(I) & =\operatorname{tr}\left(F_{\omega} \wedge *\left(F_{\omega}\right)\right)+2 \partial^{\mu} \phi \partial_{\mu} \phi \sqrt{|g|} d^{5} x \\
(I I) & :=\operatorname{tr}\left((D H)_{\mu}(D H)^{\mu \dagger}\right) \sqrt{|g|} d^{5} x \\
(I I I) & :=(D H)_{\mu}^{\dagger}(D H)^{\mu} \sqrt{|g|} d^{5} x \\
(I V) & :=F_{\alpha} \wedge *\left(F_{\alpha}\right)+\partial^{\mu} \phi \partial_{\mu} \phi \sqrt{|g|} d^{5} x .
\end{aligned}
$$

and $d^{5} x=d^{4} x \xi_{1}$. Using the fact that $\operatorname{tr}\left(A B^{\dagger}\right)=B^{\dagger} A$ for any $A, B \in M_{2 \times 1}(\mathbb{C})$, we finally find that

$$
S=f\left[\operatorname{tr}\left(F_{\omega} \wedge *\left(F_{\omega}\right)\right)+F_{\alpha} \wedge *\left(F_{\alpha}\right)+2(D H)_{\mu}^{\dagger}(D H)^{\mu} \sqrt{|g|} d^{5} x+3 \partial^{\mu} \phi \partial_{\mu} \phi \sqrt{|g|} d^{5} x\right]
$$


i.e., after dimensional reduction,

$$
S=\int_{M}\left[\operatorname{tr}\left(F_{\omega} \wedge *\left(F_{\omega}\right)_{4}\right)+F_{\alpha} \wedge *\left(F_{\alpha}\right)_{4}+2(D H)_{\mu}^{\dagger}(D H)^{\mu} \sqrt{|g|} d x^{4}+3 \partial^{\mu} \phi \partial_{\mu} \phi \sqrt{|g|} d^{4} x\right] .
$$

The first two terms in (54) are the Yang-Mills actions of the $U(2)$ and $U(1)$ gauge fields. To recover the classical $S U(2)$ and $U(1)$ gauge field strengths, we further impose the constraint that

$$
\operatorname{tr}(\omega)=\alpha
$$

There is no mass term and no quartic potential for the Higgs field, but such terms are gaugeinvariant and are generated under renormalization. We will elucidate why such terms are absent in section IV.

To determine the Yukawa couplings, we recall the definition of $\tilde{\mathcal{M}}(\mathcal{A})$ and note that $\Omega^{1}(\mathcal{A})_{V}$ has 5 generators. The Clifford action $c: \Omega^{1}(\mathcal{A})_{V} \rightarrow \operatorname{End}(\tilde{\mathcal{M}}(\mathcal{A}))$ is then given by

$$
\begin{aligned}
c\left(d x^{\mu}\right) & :=i \tilde{\gamma}^{\mu} \otimes 1 \\
c\left(\xi_{1}\right) & :=\gamma^{5} \otimes 1
\end{aligned}
$$

where $\tilde{\gamma}^{\mu}$ 's are the Dirac matrices in curved spacetime, i.e., $\tilde{\gamma}^{\mu}=e_{a}^{\mu} \gamma^{a}$ with $\left\{\gamma^{a}, \gamma^{b}\right\}=-2 \eta^{a b}$ (we work with the signature $(-,+,+,+)), \gamma^{a \dagger}=-\gamma^{a}$ for $a=1,2,3$ and $\gamma^{0 \dagger}=\gamma^{0} ; \gamma^{5}$ is given by the product $\gamma^{5}=i \gamma^{0} \gamma^{1} \gamma^{2} \gamma^{3}$. One checks that $\gamma^{5 \dagger}=\gamma^{5},\left(\gamma^{5}\right)^{2}=1$. The Dirac Operator is given by

$$
D_{c}:=c \circ \tilde{\nabla}: \tilde{\mathcal{M}}(\mathcal{A}) \rightarrow \tilde{\mathcal{M}}(\mathcal{A}) \text {. }
$$

This yields

$$
D_{c}=i \gamma^{a} e_{a}^{\mu}\left(\partial_{\mu}-\frac{1}{2} i \omega_{\mu}^{b c} \Sigma_{b c}+\Omega_{\mu}\right)+\gamma^{5} \Omega_{4}
$$

where $\Sigma_{a b}=\frac{i}{4}\left[\gamma_{a}, \gamma_{b}\right]$ and $\omega_{\mu}^{b c}$ are the components of the spin connection. We use the notations

$$
\Psi=\left(\begin{array}{c}
\psi_{1 L} \\
\psi_{2 L} \\
\psi_{3 R}
\end{array}\right), \quad H=\left(\begin{array}{c}
H_{1} \\
H_{2}
\end{array}\right), \quad \bar{\psi}_{i}=\psi_{i}^{\dagger} \gamma^{0} .
$$

The fermionic action, defined by

$$
S=\int d^{4} x \sqrt{|g|} \bar{\Psi} D_{c} \Psi
$$

for arbitrary $\Psi \in \tilde{\mathcal{M}}(\mathcal{A})$, with $\bar{\Psi}=\left(\bar{\psi}_{1 L} \bar{\psi}_{2 L} \bar{\psi}_{3 R}\right)$, gives rise to the Yukawa and axion-fermion 
couplings through the term $\bar{\Psi} \gamma^{5} \Omega_{4} \Psi$ :

$$
\begin{aligned}
\bar{\Psi} \gamma^{5} \Omega_{4} \Psi & =\left(\bar{\psi}_{1 L} H_{1} \gamma^{5} \psi_{3 R}+\bar{\psi}_{2 L} H_{2} \gamma^{5} \psi_{3 R}-\bar{\psi}_{3 R} \bar{H}_{1} \gamma^{5} \psi_{1 L}-\bar{\psi}_{3 R} \bar{H}_{2} \gamma^{5} \psi_{2 L}\right)+i \sum_{i=1}^{3} \bar{\psi}_{i} \phi \gamma^{5} \psi_{i} \\
& =-\left(\bar{\psi}_{1 L} H_{1} \psi_{3 R}+\bar{\psi}_{2 L} H_{2} \psi_{3 R}+\bar{\psi}_{3 R} \bar{H}_{1} \psi_{1 L}+\bar{\psi}_{3 R} \bar{H}_{2} \psi_{2 L}\right)+i \sum_{i=1}^{3} \bar{\psi}_{i} \phi \gamma^{5} \psi_{i} .
\end{aligned}
$$

Coupling constants can be introduced by rescaling the fields.

\section{REMARKS AND CONCLUSIONS}

We should explain why we do not find any quartic and quadratic terms for the Higgs field. For this purpose, we outline a parallel between our point of view and Connes' point of view of noncommutative geometry. To do so, we introduce the same toy model as Connes did in [6], (p.563-567). We consider the discrete space $X=\{a, b\}$, formed by two separate points. Suppose that there is a complex vector space $W_{a}$ of dimension $n_{a}$ attached to $a$, and a complex vector space $W_{b}$ of dimension $n_{b}$ attached to $b$. $W=W_{a} \oplus W_{b}$ is a projective finitely generated left $\mathbb{C} \oplus \mathbb{C}$-module, and one could, exactly as in [6], consider the algebra $\mathcal{A}=\mathbb{C} \oplus \mathbb{C}$ and introduce a connection. In Connes' formalism, the space of "noncommutative" one forms $\Omega^{1}(\mathcal{A})$ is 2-dimensional. This is the reason why his Yang-Mills action (p.567 of [6]) exhibits quartic and quadratic terms that mimic the Higgs potential.

In our approach, we consider the vector bundle $W$ as a free $\mathbb{C}$-module. We can choose a basis of sections $a_{1}, \ldots, a_{n_{a}}, b_{1}, \ldots, b_{n_{b}}$ with $a_{i} \in W_{a}$ and $b_{j} \in W_{b}$ for all $i, j$. A connection is a linear map that determines the variation of this basis when one moves along space-time. Here it quantifies the variation due to jumping from $a$ to $b$. To quantify this jump, we introduce the one-dimensional vector space $V=\left\{\lambda \xi_{1}, \lambda \in \mathbb{C}\right\}$ spanned by $\xi_{1}$, where $\xi_{1}$ plays the role of $d x$ in the direction of the jump. The variation $\Delta a_{i}=a_{i}^{\prime}-a_{i}$ can be written $\Delta a_{i}=\phi_{i 1} b_{1}+\ldots+\phi_{i n_{b}} b_{n_{b}}$, with $\phi_{i j} \in \mathbb{C}$. In the same way, $\Delta b_{i}=\phi_{i 1}^{\prime} a_{1}+\ldots+\phi_{i n_{a}}^{\prime} a_{n_{a}}$, with the $\phi_{i j}^{\prime} \in \mathbb{C}$. The connection has the form

$$
\nabla\left(\begin{array}{c}
a_{1} \\
. . \\
a_{n_{a}} \\
b_{1} \\
. . \\
b_{n_{b}}
\end{array}\right)=\left(\begin{array}{cccccc}
0 & \ldots & 0 & \phi_{11} & \ldots & \phi_{1 n_{b}} \\
\ldots & \ldots & \ldots & \ldots & \ldots & \ldots \\
0 & \ldots & 0 & \phi_{n_{a} 1} & \ldots & \phi_{n_{a} n_{b}} \\
\phi_{11}^{\prime} & \ldots & \phi_{1 n_{a}}^{\prime} & 0 & . . & 0 \\
\ldots & \ldots & \ldots & \ldots & \ldots & \ldots \\
\phi_{n_{a} 1}^{\prime} & \ldots & \phi_{n_{b} n_{a}}^{\prime} & 0 & \ldots & 0
\end{array}\right) \xi_{1} \otimes\left(\begin{array}{c}
a_{1} \\
. . \\
a_{n_{a}} \\
b_{1} \\
. . \\
b_{n_{b}}
\end{array}\right) .
$$

Our approach is thus different from Connes' approach and leads to different results. For instance, quartic and quadratic terms in the $\phi_{i j}$ 's vanish because $\Omega(\mathcal{B})_{V}$ is one-dimensional. To get such terms one must enlarge $V$, e.g. take a two-dimensional vector space. We have carried out such generalizations for the Higgs field but they lead to fermion doubling. Indeed, if $V$ has two generators 
$\xi_{1}$ and $\xi_{2}$, one can write the connection $\Omega$ of the last section in the form (neglecting the axion field)

$$
\Omega=\left(\begin{array}{cc}
\omega_{2 \times 2} & 0_{2 \times 1} \\
0_{1 \times 2} & \alpha_{1 \times 1}
\end{array}\right) \otimes 1+\left(\begin{array}{cc}
0_{2 \times 2} & H \\
-H^{\dagger} & 0
\end{array}\right) \otimes \xi_{1}+\left(\begin{array}{cc}
0_{2 \times 2} & H^{\prime} \\
-H^{\prime \dagger} & 0
\end{array}\right) \otimes \xi_{2}
$$

where $H^{\prime}=i H$ if one wants to recover a quartic term for the Higgs field in the action. Then the Clifford action $c: \Omega^{1}(\mathcal{A})_{V} \rightarrow \operatorname{End}(\tilde{\mathcal{M}}(\mathcal{A}))$ is given by

$$
\begin{aligned}
c\left(d x^{\mu}\right) & :=\Gamma^{\mu} \otimes 1 \\
c\left(\xi_{1}\right) & :=\Gamma^{5} \otimes 1 \\
c\left(\xi_{2}\right) & :=\Gamma^{6} \otimes 1
\end{aligned}
$$

where $\Gamma^{A}, A \in\{0,1,2,3,5,6\}$, are $8 \times 8$ complex matrices. The number of spinors has to be multiplied by a factor of two to make sense of $\Gamma^{A} \psi$, and we end up with fermion doubling. As there is, a priori, no obstruction against adding gauge-invariant terms to the action, we prefer a five dimensional model.

The introduction of right-handed neutrinos is possible within our formalism. The see-saw mechanism ( see [1], [12] for reviews) furnishes a potential explanation of the origin of the mass of the left-handed neutrinos of the Standard Model. It is based on the presence, in the action, of a Majorana mass term for the right-handed neutrinos, of the form $M_{r r} \bar{\nu}_{r} \nu_{r}^{c}$, and a small Dirac mass $m_{l r} \bar{\nu}_{l} \nu_{r}+$ h.c., with $m_{l r}<<M_{r r}$, coming from Yukawa couplings. The mass matrix can be written in the form

$$
\left(\bar{\nu}_{l} \bar{\nu}_{r}^{c}\right)\left(\begin{array}{cc}
0 & m_{l r} \\
m_{l r}^{\dagger} & M_{r r}
\end{array}\right)\left(\begin{array}{c}
\nu_{l}^{c} \\
\nu_{r}
\end{array}\right)
$$

The diagonalization of this mass matrix leads to a small mass for the left-handed neutrinos, of the order of $m_{l r} M_{r r}^{-1} m_{l r}^{\dagger}$, whereas the Majorana masses for the right handed neutrinos are left essentially unchanged. We can introduce a Dirac mass in our model. Consider a toy model, where we only add one right-handed neutrino, described by a Majorana spinor $\nu_{R}^{c}=\nu_{R}$. On the free $\mathcal{C}^{\infty}(M, \mathbb{C})$-module $\mathcal{C}^{\infty}\left(M, \mathbb{C}^{2} \oplus \mathbb{C}^{2}\right)$, we can choose the connection

$$
\Omega=\left(\begin{array}{cc}
\omega_{2 \times 2} & 0_{2 \times 2} \\
0_{2 \times 2} & \left(\begin{array}{ll}
\alpha & 0 \\
0 & 0
\end{array}\right)
\end{array}\right) \otimes 1+\left(\begin{array}{cc}
0_{2 \times 2} & -m_{l r} \\
m_{l r}^{\dagger} & 0
\end{array}\right) \otimes \xi_{1} .
$$

This connection leads to a Dirac mass term in the action. 


\section{Acknowledgments}

A. H. C is supported in part by the National Science Foundation under Grant No. Phys-0854779.

[1] A. Boyarsky, O. Ruchayskiy, and M. Shaposhnikov. The role of sterile neutrinos in cosmology and astrophysics. Annu. Rev. Nucl. Part. S., 59(1):191-214, 2009.

[2] A.H. Chamseddine, A. Connes, and M. Marcolli. Gravity and the standard model with neutrino mixing. Adv. Theor. Math. Phys., 11:991-1089, 2007.

[3] A.H. Chamseddine, G. Felder, and J. Fröhlich. Unified gauge theories in noncommutative geometry. Phys. Lett. B, 296(1):109-116, 1992.

[4] A.H. Chamseddine, J. Fröhlich, and O. Grandjean. The gravitational sector in the connes-lott formulation of the standard model. J. Math. Phys., 36:6255-6275, 1995.

[5] Y. Choquet-Bruhat. General Relativity and the Einstein Equations. Oxford Univ. Press, Oxford, 2009.

[6] A. Connes. Noncommutative Geometry. Academic Press, San Diego, CA, 1994.

[7] A. Connes and M. Marcolli. Noncommutative Geometry, Quantum Fields and Motives, volume 55. Amer. Math. Soc. Coll. Publ., 2008.

[8] T. Damour, G.W. Gibbons, and C. Gundlach. Dark matter, time-varying g, and a dilaton field. Phys. Rev. Lett., 64(2):123-126, 1990.

[9] J. Fröhlich, O. Grandjean, and A. Recknagel. Supersymmetric quantum theory and differential geometry. Commun. Math. Phys., 193(3):527-594, 1998.

[10] J. Fröhlich and B. Pedrini. New applications of the chiral anomaly. Mathematical physics 2000, pages 9-47, 2000.

[11] T. Kaluza. Zum Unitätsproblem der Physik. Sitz. Preuss. Akad. Wiss. Phys. Math. K, 1:966-972, 1921.

[12] S.F. King. Neutrino mass models. Rep. Prog. Phys., 67:107-157, 2004.

[13] O. Klein. Quantentheorie und fünfdimensionale Relativitätstheorie. Z. Phys. A-Hadron Nucl., 37(12):895-906, 1926.

[14] M.L. Michelson and H.B. Lawson. Spin Geometry. Princeton Univ. Press, Princeton, 1990.

[15] L. O'Raifeartaigh and N. Straumann. Gauge theory: Historical origins and some modern developments. Rev. Mod. Phys., 72(1):1-23, 2000.

[16] R.G. Swan. Vector bundles and projective modules. T. Am. Math. Soc., 105(2):264-277, 1962.

[17] S. Weinberg and O.W. Greenberg. The Quantum Theory of Fields, Vol. II: Modern Applications. Cambridge Univ. Press, Cambridge, 1996. 